\title{
A general model of multivalent binding with ligands of heterotypic subunits and multiple surface receptors
}

\author{
Zhixin Cyrillus Tan ${ }^{\mathrm{a}}$, Aaron S. Meyer ${ }^{\mathrm{a}, \mathrm{b}, \mathrm{c}, \mathrm{d}, *}$ \\ ${ }^{a}$ Bioinformatics Interdepartmental Program, University of California, Los Angeles, Los \\ Angeles, California, 90095, United States \\ ${ }^{b}$ Department of Bioengineering, University of California, Los Angeles, Los \\ Angeles, California, 90095, United States \\ ${ }^{c}$ Jonsson Comprehensive Cancer Center, University of California, Los Angeles, Los \\ Angeles, California, 90095, United States \\ ${ }^{d}$ Eli and Edythe Broad Center of Regenerative Medicine and Stem Cell Research, University \\ of California, Los Angeles, Los Angeles, California, 90095, United States
}

\begin{abstract}
Multivalent cell surface receptor binding is a ubiquitous biological phenomenon with functional and therapeutic significance. Predicting the amount of ligand binding for a cell remains an important question in computational biology as it can provide great insight into cell-to-cell communication and rational drug design toward specific targets. In this study, we extend a mechanistic, two-step multivalent binding model. This model predicts the behavior of a mixture of different multivalent ligand complexes binding to cells expressing various types of receptors. It accounts for the combinatorially large number of interactions between multiple ligands and receptors, optionally allowing a mixture of complexes with different valencies and complexes that contain heterogeneous ligand units. We derive the macroscopic predictions and demonstrate how this model enables large-scale predictions on mixture binding and the binding space of a ligand. This model thus provides an elegant and computationally efficient framework for analyzing multivalent binding.

Keywords: General Binding Model, Multivalent Binding, Combinatorics, Receptor-Ligand Interactions, Cell Surface Reactions, Protein-Protein Interactions
\end{abstract}

\section{Introduction}

Binding to extracellular ligands is among the most fundamental and universal activities of a cell. Many important biological activities, and cell-to-cell communication in particular, are based on recognizing extracellular molecules ${ }_{5}$ via specific surface receptors. For example, multivalent ligands are common

\footnotetext{
* Corresponding author

Email address: ameyer@asmlab.org (Aaron S. Meyer)
} 
extracellular factors in the immune system [1, and computational models have been applied to study IgE-FceRI [2, MHC-T cell receptor [3], and IgG-Fc $\gamma \mathrm{R}$ interactions [4]. However, these models are specific to their biological applications, limited to a single homogenous ligand and receptor [5], or fail to scale ith valency [6].

Multivalent binding to various receptors on a cell can be accounted for by the kinetics of individual association reactions between each monomer-receptor pair. However, when the complexes contain multiple ligand monomers of either the same or different kinds, and when there is a mixture of complexes with ei-

15 ther the same or different valencies, the system becomes complicated: different binding orders of units on a complex creates a combinatorially large amount of possible reactions, and the competition among different kinds of ligands and complexes impedes intuitive understanding. In this case, enumerating all binding configurations and reactions become impractical.

20 cases involving multiple receptors and ligand subunits $7,8,5,5,9,3$. By harnessing the power of combinatorics via applying the multinomial theorem and focusing on macrostates, we can predict the amount of binding for each ligand and receptor at equilibrium. We derive macroscopic quantities for both specif-

25 ically arranged and randomly assorted complexes, and demonstrate how this model enables large-scale predictions on mixture binding and the binding space of a ligand.

Our model provides both generality and computational efficiency, allowing large-scale predictions such as characterizing synergism of using a mixture of so ligands and depicting the general binding behavior of a compound. The compactness and elegance of the formulae enable both analytical and numerical analyses, in turn allowing for the construction of higher-level computational tools. We expect this binding model will be widely applicable to many biological contexts.

\section{Preliminaries}

\subsection{Vector and matrix notation}

In this work, we denote a vector in boldface letter and its entry in the same letter but with subscript and not in boldface, e.g. $\mathbf{C}=\left[C_{1}, C_{2}, \ldots, C_{n}\right]$. The sum of elements for a vector is denoted as $|\mathbf{C}|=\sum_{i=1}^{n} C_{i}$.

40 For any matrix $\left(A_{i j}\right)$ of size $m \times n$, we denote the vector formed by its $i$-th row as $\mathbf{A}_{\mathbf{i} \bullet}=\left[A_{i 1}, A_{i 2}, \cdots, A_{i n}\right]$, and the vector formed by its $j$-th column as $\mathbf{A}_{\bullet \mathbf{j}}=\left[A_{1 j}, A_{2 j}, \cdots, A_{m j}\right]$. The row sums of matrix $\left(A_{i j}\right)$, therefore, can be written as $\left|\mathbf{A}_{\mathbf{1}}\right|,\left|\mathbf{A}_{\mathbf{2}}\right|, \cdots,\left|\mathbf{A}_{\mathbf{m} \bullet}\right|$, and column sums $\left|\mathbf{A}_{\bullet \mathbf{1}}\right|,\left|\mathbf{A}_{\bullet \mathbf{2}}\right|, \cdots,\left|\mathbf{A}_{\bullet \mathbf{n}}\right|$.

In this work, multinomial coefficients such as $n$ choose $k_{1}, k_{2}, \cdots, k_{n}$ will be 45 written as

$$
\left(\begin{array}{l}
n \\
\mathbf{k}
\end{array}\right)=\left(\begin{array}{cccc} 
& n & \\
k_{1} & k_{2} & \cdots & k_{n}
\end{array}\right)=\frac{n !}{k_{1} ! k_{2} ! \cdots k_{n} !} .
$$

The implicit assumption here is that $|\mathbf{k}|=n$, and each $k_{i} \in \mathbb{N}$. 


\subsection{Some useful theorems in combinatorics}

From the binomial theorem, we know that

$$
\sum_{i=0}^{f}\left(\begin{array}{l}
f \\
i
\end{array}\right) \Phi^{i}=(1+\Phi)^{f}
$$

Differentiating both sides by $\Phi$, we get

$$
\sum_{i=0}^{f} i\left(\begin{array}{l}
f \\
i
\end{array}\right) \Phi^{i}=f \Phi(1+\Phi)^{f-1} .
$$

We can derive similar property from the multinomial theorem. Assume the elements of a nonnegative integer vector $\mathbf{q}$ add up to $f$, or $|\mathbf{q}|=f$. Given another nonnegative vector $\varphi$ with sum of elements $|\varphi|$, we have

$$
\sum_{|\mathbf{q}|=f}\left(\begin{array}{l}
f \\
\mathbf{q}
\end{array}\right) \prod_{i} \varphi_{i}^{q_{i}}=|\varphi|^{f} .
$$

Differentiate both sides by $\varphi_{m}$ where $\varphi_{m}$ can be any entry of $\varphi$, we have

$$
\sum_{|\mathbf{q}|=f}\left(\begin{array}{l}
f \\
\mathbf{q}
\end{array}\right) q_{m} \prod_{i} \varphi_{i}^{q_{i}}=\varphi_{m} f|\varphi|^{f-1} .
$$

We can multiply two independent multinomial theorem equations together, too. Let $\mathbf{u}$ and $\mathbf{v}$ be two nonnegative integer vectors, $\mathbf{a}$ and $\mathbf{b}$ be two nonnegative vectors, and $|\mathbf{u}|=m,|\mathbf{v}|=n$, we have

$$
\sum_{\substack{|\mathbf{u}|=m \\
|\mathbf{v}|=n}}\left(\begin{array}{c}
m \\
\mathbf{u}
\end{array}\right)\left(\begin{array}{c}
n \\
\mathbf{v}
\end{array}\right) \prod_{i} a_{i}^{u_{i}} \prod_{j} b_{j}^{v_{j}}=|\mathbf{a}|^{m}|\mathbf{b}|^{n} .
$$

Throughout this work, we consolidate multiple summation symbols into one.

In this case, we use $\sum_{|\mathbf{u}|=m,|\mathbf{v}|=n}$ as a shorthand for $\sum_{|\mathbf{u}|=m} \sum_{|\mathbf{v}|=n}$. From Equation 2.5, we can derive the sum of a linear combination of two exponents from each multinomial term as

$$
\begin{aligned}
\sum_{\substack{|\mathbf{u}|=m \\
|\mathbf{v}|=n}}\left(\begin{array}{c}
m \\
\mathbf{u}
\end{array}\right)\left(\begin{array}{l}
n \\
\mathbf{v}
\end{array}\right) \underbrace{\left(k_{1} u_{p}+k_{2} v_{q}\right)}_{\text {linear combination }} \prod_{i} a_{i}^{u_{i}} \prod_{j} b_{j}^{v_{j}} & =k_{1} a_{p} m|\mathbf{a}|^{m-1}|\mathbf{b}|^{n}+k_{2}|\mathbf{a}|^{m} b_{q} n|\mathbf{b}|^{n-1} \\
& =\left[\frac{k_{1} m a_{p}}{|\mathbf{a}|}+\frac{k_{2} n b_{q}}{|\mathbf{b}|}\right]|\mathbf{a}|^{m}|\mathbf{b}|^{n}
\end{aligned}
$$

where $k_{1}$ and $k_{2}$ can be any constant.

We can extend this to the product of $N$ multinomial equations. Let $\mathbf{q}_{1}, \cdots, \mathbf{q}_{\mathbf{N}}$ be $N$ nonnegative integer vectors, each with $\left|\mathbf{q}_{\mathbf{i}}\right|=\theta_{i}$, and $\boldsymbol{\psi}_{\mathbf{1}}, \cdots, \boldsymbol{\psi}_{N}$ be $N$ 
nonnegative vectors. The sum of any linear combination of exponent terms

${ }_{65} \sum_{r} k_{r} q_{s_{r} t_{r}}$, where $k_{r}$ 's can be any constant, and each $q_{s_{r} t_{r}}$ is the $t_{r}$-th element of $\mathbf{q}_{\mathbf{s}_{\mathbf{r}}}$, can be calculated as

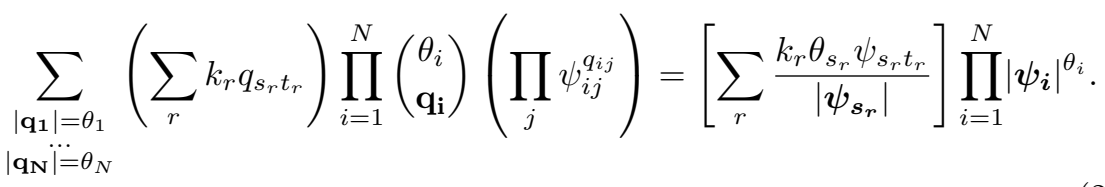

\section{Model setup}

\subsection{Parameters and notations}

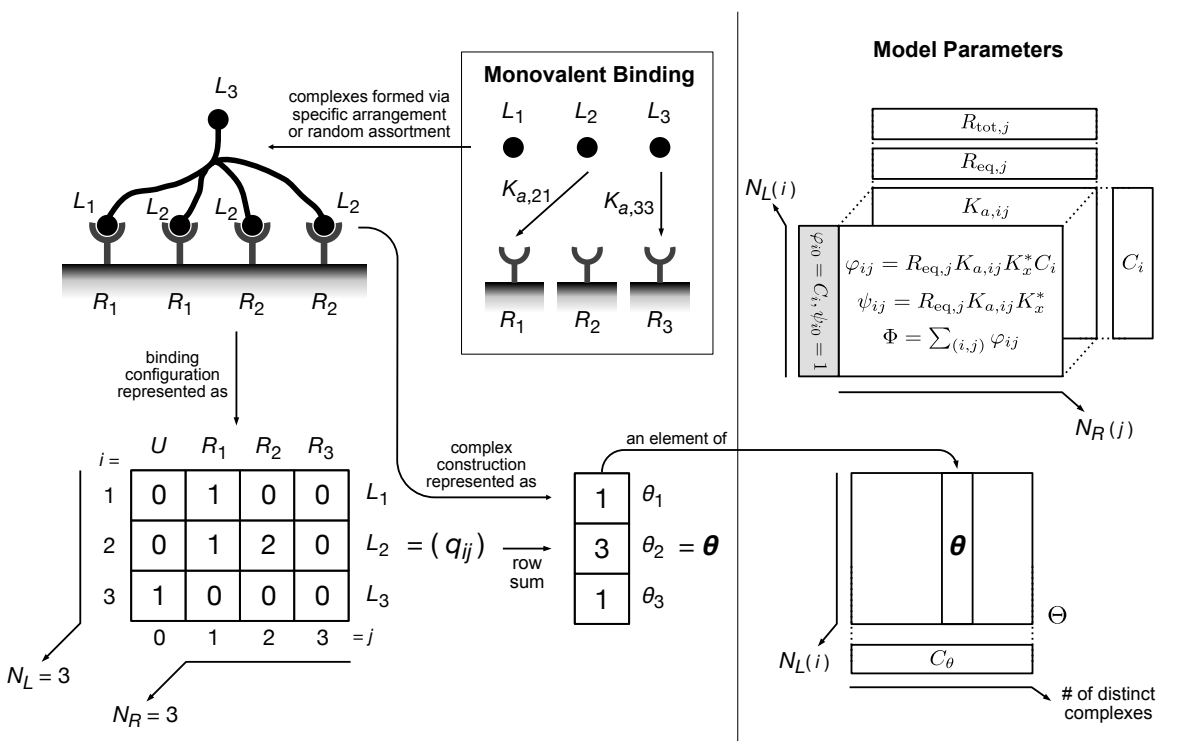

Figure 1: General setup of the model. In this study, we investigate the binding behavior of complexes formed by monomer ligands in either a specific arrangement or by random assortment. We propose that the binding configuration between a complex and several receptors on a cell can be described as a matrix $\left(q_{i j}\right)$. The construction of a complex can be written as a vector $\boldsymbol{\theta}$. The figure shows the dimensions of the model's parameters: $C_{i}$, the monomer compositions, are in a vector of length $N_{L} ; R_{\mathrm{tot}, j}$ and $R_{\mathrm{eq}, j}$, the receptor expression and equilibrium levels are in vectors of length $N_{R}$; the binding affinities, $K_{a, i j}$, are in a matrix of dimension $N_{L} \times N_{R} ; \varphi_{i j}$ and $\psi_{i j}$ are in the matrices of dimension $N_{L} \times\left(N_{R}+1\right)$. $\Theta$ is a set of all possible $\boldsymbol{\theta}$ 's, with $C_{\boldsymbol{\theta}}$ as each of their compositions. Each $\boldsymbol{\theta}$ is a vector of length $N_{L}$, and $C_{\boldsymbol{\theta}}$ should be in a vector of the same size as $\Theta$.

In this study, we investigate the binding between multivalent ligand complexes and a cell expressing various surface receptors. As shown in Figure 1, we consider $N_{L}$ types of distinct monomer ligands, namely $L_{1}, L_{2}, \ldots, L_{N_{L}}$, and $N_{R}$ types of distinct receptors expressed on a cell, namely $R_{1}, R_{2}, \ldots, R_{N_{R}}$. 
The monovalent binding association constant between $L_{i}$ and $R_{j}$ is defined as $K_{a, i j}$. A ligand complex consists of one or several monomer ligands, and each 75 of them can bind to a receptor independently. Its construction can be described by a vector $\boldsymbol{\theta}=\left[\theta_{1}, \theta_{2}, \ldots, \theta_{N_{L}}\right]$, where each entry $\theta_{i}$ represents how many $L_{i}$ this complex contains. The sum of elements of vector $\boldsymbol{\theta},|\boldsymbol{\theta}|$, is $f$, the valency of this complex.

The binding configuration at equilibrium between an individual complex and

so a cell expressing various receptors can be described as a matrix $\left(q_{i j}\right)$ with $N_{L}$ rows and $\left(N_{R}+1\right)$ columns. For example, the complex bound as shown on the top left corner in Figure 1 can be described as the matrix below it. $q_{i j}$ represents the number of $L_{i}$ to $R_{j}$ binding, and $q_{i 0}$, the entry on the 0 -th column, is the number of unbound $L_{i}$ on that complex in this configuration. This matrix can ${ }_{85}$ be unrolled into a vector form $\mathbf{q}=\left[q_{10}, q_{11}, \ldots, q_{1 N_{R}}, q_{20}, \ldots, q_{2 N_{R}}, q_{30}, \ldots, q_{N_{L} N_{R}}\right]$ of length $N_{L}\left(N_{R}+1\right)$. Note that this binding configuration matrix $\left(q_{i j}\right)$ only records how many $L_{i}$-to- $R_{j}$ pairs are formed, regardless of which exact ligand on the complex binds. For example, in Figure 1, swapping the two $L_{2}$ 's binding to $R_{2}$ 's will give us the same configuration matrix. Therefore, we will need to o account for this combinatorial factor when applying the law of mass action.

We know from the conservation of mass that for this complex, $\theta_{i}=q_{i 0}+$ $q_{i 1}+q_{i 2}+\cdots+q_{i N_{R}}=\left|\mathbf{q}_{\mathbf{i} \bullet}\right|$ must hold for all $i$ 's. Mathematically, vector $\boldsymbol{\theta}$ is the row sums of matrix $\left(q_{i j}\right)$. The corresponding $\boldsymbol{\theta}$ of a binding configuration $\mathbf{q}, \boldsymbol{\theta}(\mathbf{q})$, written in the format of a function of $\mathbf{q}$, can be determined by this 95 relationship. Also, the sum of elements in $\mathbf{q},|\mathbf{q}|=f$, will be the valency.

The concentration of complexes in the solution is $L_{0}$ (not to be confused with $L_{i}$, the name of ligands, when $\left.i=1,2, \cdots, N_{L}\right)$. It is the concentration of all ligands at the equilibrium state. It is approximately the same as the initial ligand concentration if the amount of ligands is much greater than that of the receptors and binding event does not significantly deplete the ligand concentration.

On the receptor side, $R_{\mathrm{tot}, i}$ is the total number of $R_{i}$ expressed on the cell surface. This usually can be measured experimentally. $R_{\mathrm{eq}, i}$ is the number of unbound $R_{i}$ on a cell at the equilibrium state during the ligand complex-receptor ${ }_{105}$ interaction, and usually must be calculated from $R_{\mathrm{tot}, i}$ as we will explain later.

The binding of a ligand complex, a large molecule, is complicated. To simplify the matter, we will need to make some key thermodynamic assumptions. In this model, we make two assumptions on the binding dynamics:

1. The initial binding of $L_{i}$ on a free (unbound) complex to a surface receptor $R_{j}$ has the same affinity (association constant, $K_{a, i j}$ ) as that of a monomer ligand $L_{i}$;

2. In order for the detailed balance to hold, the association constant of any subsequent binding event on the surface of a cell after the initial interaction must be proportional to their corresponding monovalent affinity. We assume the subsequent binding affinity in multivalent interactions between $L_{i}$ and $R_{j}$ to be $K_{x}^{*} K_{a, i j}$.

$K_{x}^{*}$ is a term coined as the crosslinking constant. It captures the difference 
between free and multivalent ligand-receptor binding, including but not limited to steric effects and local receptor clustering [10. In practice this term is often fit to apply this model to a specific biological context.

We create two last variables that will help to simplify our equations. For all $i$ in $\left\{1,2, \ldots, N_{L}\right\}$, we define $\psi_{i j}=R_{\mathrm{eq}, j} K_{a, i j} K_{x}^{*}$ and $\varphi_{i j}=R_{\mathrm{eq}, j} K_{a, i j} K_{x}^{*} C_{i}$ where $j=\left\{1,2, \ldots, N_{R}\right\}$, and we define $\psi_{i 0}=1, \varphi_{i 0}=C_{i}$. Therefore, $\varphi_{i j}=\psi_{i j} C_{i}$ holds for all $i$ and $j$. Then we define the sum of this new matrix $\left(\varphi_{i j}\right)$ as

${ }_{125} \sum_{i=1}^{N_{L}} \sum_{j=1}^{N_{R}} \varphi_{i j}=\Phi$, and $\sum_{i=1}^{N_{L}} \sum_{j=0}^{N_{R}} \varphi_{i j}=\Phi+\sum_{i=1}^{N_{L}} C_{i}=1+\Phi$. The rationale of these definitions will become clear in future sections.

\subsection{The amount of a specific binding configuration}

With the definitions of our model we can now derive the amount of complexes bound with the configuration described as $\mathbf{q}$ on a cell at equilibrium, $v_{\mathbf{q}}$.

We know that the composition of any complex can be described by a vector $\boldsymbol{\theta}$ of length $N_{L}$, where each entry $\theta_{i}$ represents the number of monomers $L_{i}$ within the complex. We can enumerate all possible binding configurations of $\boldsymbol{\theta}$ complex by filling the matrix $\left(q_{i j}\right)$ with any nonnegative integer values so long as its row sums equal $\boldsymbol{\theta}$. Conversely, for a certain binding configuration, $\mathbf{q}$, the construction of the complex involved must be its row sum, $\boldsymbol{\theta}(\mathbf{q})$, and the concentration of this complex is $L_{0} C_{\boldsymbol{\theta}(\mathbf{q})}$. If the corresponding complex $\boldsymbol{\theta}(\mathbf{q})$ does not exist in the solution, we set $C_{\boldsymbol{\theta}(\mathbf{q})}=0$. Since $\boldsymbol{\theta}(\mathbf{q})$ is defined only by the ligand concentration at equilibrium, it will remain $L_{0} C_{\boldsymbol{\theta}(\mathbf{q})}$.

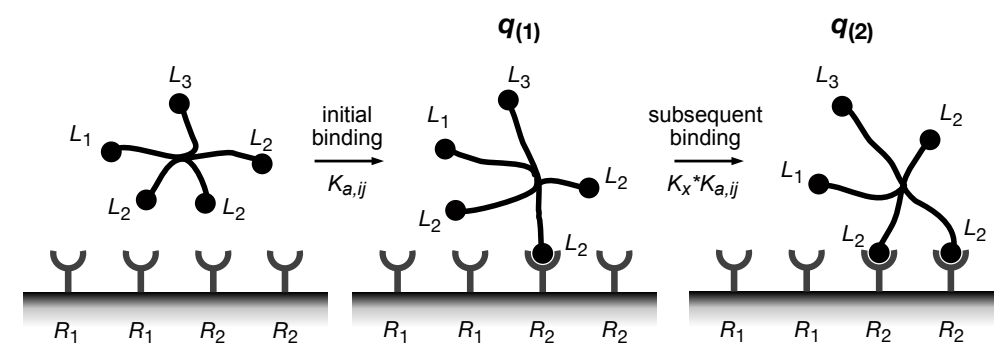

$\theta(q)$

\begin{tabular}{|l|}
\hline 1 \\
\hline 3 \\
\hline 1 \\
\hline
\end{tabular}

$q_{a}$

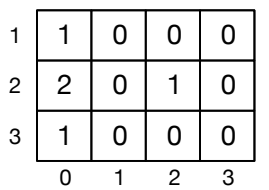

$q_{b}$

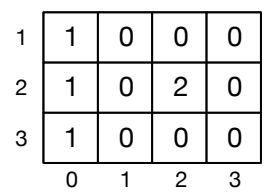

Figure 2: The scheme of cell-complex binding step by step. We assume the initial binding event has the same affinity as monomer binding, $K_{a, i j}$, while subsequent binding has an association constant scaled by $K_{x}^{*}$, the crosslinking constant. Each binding configuration scheme above can be described by the $\mathbf{q}$ right below, if we ignore the statistical factors. $\boldsymbol{\theta}(\mathbf{q})$ is the construction of the complex and can be implied from $\mathbf{q}$. 
Initial binding. We start with the initial binding reaction of a complex, $L_{i}$-to$R_{j}$. As shown in Figure 2, the reactants are the free complexes and the free receptors $R_{j}$ (in this case $R_{2}$ ), and the product are the $L_{i}$-to- $R_{j}$ (in this case $L_{2}-R_{2}$ ) monovalently bound complexes $\mathbf{q}_{(\mathbf{1})}$. We denote the amount of this new complex as $v_{\mathbf{q}_{(1)}}$. The concentration of free complexes is $L_{0} C_{\boldsymbol{\theta}\left(\mathbf{q}_{(1)}\right)}$. The equilibrium constant for this reaction is $K_{a, i j}$. Therefore, we have

$$
v_{\mathbf{q}_{(1)}}=L_{0} C_{\boldsymbol{\theta}\left(\mathbf{q}_{(1)}\right)} R_{\mathrm{eq}, j} K_{a, i j} .
$$

145

While the binding configuration of $\mathbf{q}_{(\mathbf{1})}$ can be described by $\mathbf{q}_{\mathbf{a}}$, the total amount of complexes that bind as described as $\mathbf{q}_{\mathbf{a}}$ may not be the same as $v_{\mathbf{q}_{(1)}}$, since $\mathbf{q}_{\mathbf{a}}$ does not consider the number of ways this binding $L_{i}$ can be chosen. An equivalent explanation is that, $\mathbf{q}_{(\mathbf{1})}$ is only one possible microstate to achieve the $\mathbf{q}_{\mathbf{a}}$ configuration, and we need to count the total number of possible microstates for $\mathbf{q}_{\mathbf{a}}$. Accounting for this statistical factor, we have

$$
\begin{gathered}
v_{\mathbf{q}_{\mathbf{a}}}=v_{\mathbf{q}_{(\mathbf{1})}}\left(\begin{array}{c}
\theta_{i} \\
1
\end{array}\right)=v_{\mathbf{q}_{(1)}}\left(\begin{array}{c}
\theta_{i} \\
\mathbf{q}_{\mathbf{a}, \mathbf{i} \bullet}
\end{array}\right), \\
v_{\mathbf{q}_{\mathbf{a}}}=L_{0} C_{\boldsymbol{\theta}\left(\mathbf{q}_{\mathbf{a}}\right)} R_{\mathrm{eq}, j} K_{a, i j}\left(\begin{array}{c}
\theta_{i} \\
\mathbf{q}_{\mathbf{a}, \mathbf{i} \bullet}
\end{array}\right),
\end{gathered}
$$

since $\boldsymbol{\theta}\left(\mathbf{q}_{(\mathbf{1})}\right)=\boldsymbol{\theta}\left(\mathbf{q}_{\mathbf{a}}\right)$. $\mathbf{q}_{\mathbf{a}, \mathbf{i}}$ is a vector formed by the $i$-th row of $\mathbf{q}_{\mathbf{a}}$. For example, in Figure 2, $\mathbf{q}_{\mathbf{a}, \mathbf{2}} \bullet=[2,0,1,0]$. Conceptually, $\left(\begin{array}{c}\theta_{i} \\ \mathbf{q}_{\mathbf{a}, \mathbf{i}}\end{array}\right)$ can be understood as the number of ways to split $\theta_{i} L_{i}$ 's into $q_{i 0}$ of unbound, $q_{i 1}$ of $R_{1}$-bound, $q_{i 2}$ of $R_{2}$-bound, ..., and $q_{i N_{R}}$ of $R_{N_{R}}$-bound units. In the initial binding reaction, only $q_{i 0}$ and $q_{i j}$ will be nonzero, with $q_{i 0}=\theta_{i}-1$ and $q_{i j}=1$, so it is effectively the same as $\left(\begin{array}{c}\theta_{i} \\ 1\end{array}\right)$. However, the multinomial coefficient expression can be generalized to subsequent binding steps.

Subsequent binding. For a subsequent binding between $L_{i}$ and $R_{j}(i$ and $j$ are not necessarily the same as in initial binding), we have the reactants as a bound complex, $\mathbf{q}_{(\mathbf{1})}$, and a free receptor $R_{j}$ (in the case shown by Figure $2, R_{2}$ ), while the product is another bound complex, $\mathbf{q}_{(\mathbf{2})}$. The equilibrium constant is $K_{x}^{*} K_{a, i j}$, then

$$
v_{\mathbf{q}_{(\mathbf{2})}}=v_{\mathbf{q}_{(1)}} R_{\mathrm{eq}, j} K_{x}^{*} K_{a, i j} .
$$

To account for the statistical factors of $v_{\mathbf{q}_{\mathbf{b}}}$, we have $v_{\mathbf{q}_{\mathbf{b}}}=v_{\mathbf{q}_{(\mathbf{2})}}\left(\begin{array}{c}\theta_{i} \\ \mathbf{q}_{\mathbf{b}, \mathbf{i}}\end{array}\right)$. For example, in Figure 2, $\mathbf{q}_{\mathbf{b}, \mathbf{2}} \bullet=[1,0,2,0]$. Putting these together, we have

$$
v_{\mathbf{q}_{\mathbf{b}}}=v_{\mathbf{q}_{\mathbf{a}}} R_{\mathrm{eq}, j} K_{x}^{*} K_{a, i j} \frac{\left(\begin{array}{c}
\theta_{i} \\
\mathbf{q}_{\mathbf{b}, \mathbf{i}}
\end{array}\right)}{\left(\begin{array}{c}
\theta_{i} \\
\mathbf{q}_{\mathbf{a}, \mathbf{i}}
\end{array}\right)} .
$$

By recursion, we can solve $v_{\mathbf{q}}$ for any $\mathbf{q}$ from these equations. It is 


$$
\begin{aligned}
v_{\mathbf{q}} & =\frac{L_{0} C_{\boldsymbol{\theta}(\mathbf{q})}}{K_{x}^{*}} \prod_{(i, j)=(1,1)}^{\left(N_{L}, N_{R}\right)}\left(R_{\mathrm{eq}, j} K_{x}^{*} K_{a, i j}\right)^{q_{i j}} \prod_{i=1}^{N_{L}}\left(\begin{array}{c}
\theta_{i} \\
\mathbf{q}_{\mathbf{i} \bullet}
\end{array}\right) \\
& =\frac{L_{0} C_{\boldsymbol{\theta}(\mathbf{q})}}{K_{x}^{*}} \prod_{(i, j)=(1,1)}^{\left(N_{L}, N_{R}\right)} \psi_{i j}^{q_{i j}} \prod_{i=1}^{N_{L}}\left(\begin{array}{c}
\theta_{i} \\
\mathbf{q}_{\mathbf{i} \bullet}
\end{array}\right) \\
& =\frac{L_{0} C_{\boldsymbol{\theta}(\mathbf{q})}}{K_{x}^{*}} \prod_{(i, j)=(1,0)}^{\left(N_{L}, N_{R}\right)} \psi_{i j}^{q_{i j}} \prod_{i=1}^{N_{L}}\left(\begin{array}{c}
\theta_{i} \\
\mathbf{q}_{\mathbf{i} \bullet}
\end{array}\right)
\end{aligned}
$$

if we define $\psi_{i j}=R_{\mathrm{eq}, j} K_{x}^{*} K_{a, i j}$ for $j=1,2, \cdots, N_{R}$ and $\psi_{i 0}=1$ for all $i$. $\prod_{(i, j)=(1,0)}^{\left(N_{L}, N_{R}\right)}$ is a shorthand for $\prod_{i=1}^{N_{L}} \prod_{j=0}^{N_{R}}$. In the next section, we will use this formula repeatedly.

Notice that this equation is only for surface-bound complexes, not suitable for calculating the concentration of unbound $\mathbf{q}$, when every nonzero values are on its 0-th column. The concentration of unbound ligands should always be $L_{0} C_{\boldsymbol{\theta}(\mathbf{q})}$. However, for algebraic convenience, we allow such definition but only to subtract them later, and will name it $v_{0, \text { eq }}$ which equals $L_{0} C_{\boldsymbol{\theta}(\mathbf{q})} / K_{x}^{*}$.

\section{Macroscopic equilibrium predictions}

From here we will investigate the macroscopic properties of binding, such as the total amount of ligand bound and receptor bound on a cell surface at equilibrium. We consider two different ways complexes in the solution can be formed. First, complexes may come in a specific arrangement. In this case, the structure and exact concentration for each complex are designed and known. plexes with a fixed valency $f$. Through random assortment, any combination of $f$ monomer ligands can form a complex, and their concentration will follow a multinomial distribution. We will explore these two cases separately.

\subsection{Complexes formed in a specific arrangement}

185

When complexes are specifically arranged, the structure and proportion of each kind are well-defined. To formulate this mathematically, we assume that we have various kinds of complexes, and each of them can be described by a vector $\boldsymbol{\theta}$ of length $N_{L}$, with each entry $\theta_{i}$ as the number of $L_{i}$ in this complex. The valency of each complex may be different, and for complex $\boldsymbol{\theta}$ its valency is $|\boldsymbol{\theta}|$. The proportion of $\boldsymbol{\theta}$ among all complexes is defined as $C_{\boldsymbol{\theta}}$, and the concentration of each $\boldsymbol{\theta}$ complex will be $L_{0} C_{\boldsymbol{\theta}}$. For example, if we create a mixture of $20 \%$ of bivalent $L_{1}$ and $80 \%$ of bispecific $L_{1}-L_{2}$, then $\boldsymbol{\theta}_{\mathbf{1}}=[2,0], \boldsymbol{\theta}_{\mathbf{2}}=[1,1]$, $C_{\boldsymbol{\theta}_{1}}=20 \%$, and $C_{\boldsymbol{\theta}_{2}}=80 \%$. If the mixture solution has a total concentration of $10 \mathrm{nM}$, then the concentration of $\boldsymbol{\theta}_{\mathbf{1}}$ is $2 \mathrm{nM}$, and the concentration of $\boldsymbol{\theta}_{\mathbf{2}}$ is $8 \mathrm{nM}$. 
We further conceptualize that $\Theta$ is a set of all existing $\boldsymbol{\theta}$ 's. By this setting, we should have $\sum_{\boldsymbol{\theta} \in \Theta} C_{\boldsymbol{\theta}}=1$. These complexes will bind in various configurations which can all be described as a $\mathbf{q}$. We define $Q$ as a set of all possible q's, and we borrow the notation $\mathbf{q} \subseteq \boldsymbol{\theta}$ to indicate any binding configuration $\mathbf{q}$ that can be achieved by complex $\boldsymbol{\theta}$. This is equivalent to $\left|\mathbf{q}_{\mathbf{i}} \boldsymbol{|}\right|=\theta_{i}$ for all $i$, or $\boldsymbol{\theta}$ is the row sum of $\left(q_{i j}\right)$.

Solve the amount of free receptors. A remaining problem in the model setup is that in practice, only $R_{\mathrm{tot}, j}$, the total receptor expressions of each kind of a cell, can be experimentally measured, while the amount of free receptors at equilibrium, $R_{\mathrm{eq}, j}$, though being used extensively, is unknown. To find $R_{\mathrm{eq}, j}$, we first need to derive the amount of bound receptors of each kind, $R_{\text {bound, } j \text {, }}$ then use conservation of mass to solve $R_{\mathrm{eq}, j}$ numerically.

To calculate the amount of bound ligand $R_{\text {bound, } n}$, we can simply add up all entries in the $n$-th column for every q's:

$$
\begin{aligned}
R_{\text {bound }, n} & =\sum_{\mathbf{q} \in Q}|\mathbf{q} \bullet \mathbf{n}| v_{\mathbf{q}} \\
& =\sum_{\mathbf{q} \in Q}|\mathbf{q} \bullet \mathbf{n}| \frac{L_{0} C_{\boldsymbol{\theta}(\mathbf{q})}}{K_{x}^{*}} \prod_{(i, j)=(1,0)}^{\left(N_{L}, N_{R}\right)} \psi_{i j}^{q_{i j}} \prod_{i=1}^{N_{L}}\left(\begin{array}{c}
\theta_{i} \\
\mathbf{q}_{\mathbf{i} \bullet}
\end{array}\right) \\
& =\frac{L_{0}}{K_{x}^{*}} \sum_{\boldsymbol{\theta} \in \Theta} C_{\boldsymbol{\theta}} \sum_{\mathbf{q} \subseteq \boldsymbol{\theta}}|\mathbf{q} \bullet \mathbf{n}| \prod_{(i, j)=(1,0)}^{\left(N_{L}, N_{R}\right)} \psi_{i j}^{q_{i j}} \prod_{i=1}^{N_{L}}\left(\begin{array}{c}
\theta_{i} \\
\mathbf{q}_{\mathbf{i} \bullet}
\end{array}\right) \\
& =\frac{L_{0}}{K_{x}^{*}} \sum_{\boldsymbol{\theta} \in \Theta} C_{\boldsymbol{\theta}}\left[\frac{\psi_{1 n}}{\left|\boldsymbol{\psi}_{\mathbf{1}}\right|} \theta_{1}+\cdots+\frac{\psi_{N_{L} n}}{\mid \boldsymbol{\psi}_{N_{L}} \bullet} \theta_{N_{L}}\right] \prod_{i=1}^{N_{L}}\left|\boldsymbol{\psi}_{\boldsymbol{i}} \bullet\right|^{\theta_{i}} \\
& =\frac{L_{0}}{K_{x}^{*}} \sum_{\boldsymbol{\theta} \in \Theta} C_{\boldsymbol{\theta}}\left[\sum_{i=1}^{N_{L}} \frac{\psi_{i n}}{\left|\boldsymbol{\psi}_{\boldsymbol{i}}\right|} \theta_{i}\right] \prod_{i=1}^{N_{L}}\left|\boldsymbol{\psi}_{\boldsymbol{i}}\right|^{\theta_{i}},
\end{aligned}
$$

where $\left|\mathbf{q}_{\bullet \mathbf{n}}\right|=\sum_{m=1}^{N_{L}} q_{m n}$, and $\left|\boldsymbol{\psi}_{\boldsymbol{i}}\right|=\sum_{j=0}^{N_{R}} \psi_{i j}$.

By the conservation of mass, we have

$$
\begin{aligned}
R_{\mathrm{tot}, n} & =R_{\mathrm{eq}, n}+R_{\mathrm{bound}, n} \\
& =R_{\mathrm{eq}, n}+\frac{L_{0}}{K_{x}^{*}} \sum_{\boldsymbol{\theta} \in \Theta} C_{\boldsymbol{\theta}}\left[\sum_{i=1}^{N_{L}} \frac{\psi_{i n}}{\left|\boldsymbol{\psi}_{\boldsymbol{i}}\right|} \theta_{i}\right] \prod_{i=1}^{N_{L}}\left|\boldsymbol{\psi}_{\boldsymbol{i}}\right|^{\theta_{i}}
\end{aligned}
$$

In this equation, $R_{\mathrm{tot}, n}$ are known, and any $\left|\boldsymbol{\psi}_{\boldsymbol{i}}\right|$ is a function of every $R_{\mathrm{eq}, j}, j=1,2, \cdots, N_{R}$, so all $R_{\mathrm{eq}, j}$ need to be solved together. This system of equations usually does not have a closed form and must be solved numerically. When implementing, we suggest taking the logarithm of both sides of these equations so the exponents can be eliminated and the range is restricted to positive numbers. 
As a side note, the total amount of bound receptors regardless of kind is

$$
\begin{aligned}
R_{\text {bound }} & =\sum_{n=1}^{N_{R}} R_{\text {bound }, n} \\
& =\frac{L_{0}}{K_{x}^{*}} \sum_{\boldsymbol{\theta} \in \Theta} C_{\boldsymbol{\theta}} \sum_{n=1}^{N_{R}}\left[\frac{\psi_{1 n}}{\left|\boldsymbol{\psi}_{\mathbf{1} \bullet}\right|} \theta_{1}+\cdots+\frac{\psi_{N_{L} n}}{\left|\boldsymbol{\psi}_{\boldsymbol{N}_{L} \bullet}\right|} \theta_{N_{L}}\right] \prod_{i=1}^{N_{L}}\left|\boldsymbol{\psi}_{\boldsymbol{i}}\right|^{\theta_{i}} \\
& =\frac{L_{0}}{K_{x}^{*}} \sum_{\boldsymbol{\theta} \in \Theta} C_{\boldsymbol{\theta}}\left[\left(1-\frac{\psi_{10}}{\left|\boldsymbol{\psi}_{\mathbf{1} \bullet}\right|}\right) \theta_{1}+\cdots+\left(1-\frac{\psi_{N_{L} 0}}{\left|\boldsymbol{\psi}_{N_{L} \bullet}\right|}\right) \theta_{N_{L}}\right] \prod_{i=1}^{N_{L}}\left|\boldsymbol{\psi}_{\boldsymbol{i}}\right|^{\theta_{i}} \\
& =\frac{L_{0}}{K_{x}^{*}} \sum_{\boldsymbol{\theta} \in \Theta} C_{\boldsymbol{\theta}}\left[|\boldsymbol{\theta}|-\sum_{i=1}^{N_{L}} \frac{\theta_{i}}{\left|\boldsymbol{\psi}_{\boldsymbol{i}}\right|}\right] \prod_{i=1}^{N_{L}}\left|\boldsymbol{\psi}_{\boldsymbol{i} \bullet}\right|^{\theta_{i}} .
\end{aligned}
$$

The amount of bound ligand complexes. Our model makes many macroscopic predictions readily accessible. For example, the amount of ligand bound at equilibrium is a useful quantity when measuring the overall quantity of tagged ligand. To compute this number, we can add up all $v_{\mathbf{q}}$ except the q's that only have nonzero values on the 0 -th column, $v_{0, \text { eq }}$. Consequently, the model prediction of bound ligand at equilibrium is

$$
\begin{aligned}
& L_{\text {bound }}=\sum_{\mathbf{q} \in Q} v_{\mathbf{q}}-v_{0, \mathrm{eq}} \\
& =\sum_{\mathbf{q} \in Q} \frac{L_{0} C_{\boldsymbol{\theta}(\mathbf{q})}}{K_{x}^{*}} \prod_{(i, j)=(1,0)}^{\left(N_{L}, N_{R}\right)} \psi_{i j}{ }^{q_{i j}} \prod_{i=1}^{N_{L}}\left(\begin{array}{c}
\theta_{i} \\
\mathbf{q}_{\mathbf{i} \bullet}
\end{array}\right)-\frac{L_{0}}{K_{x}^{*}} \sum_{\boldsymbol{\theta} \in \Theta} C_{\boldsymbol{\theta}} \\
& =\frac{L_{0}}{K_{x}^{*}} \sum_{\boldsymbol{\theta} \in \Theta} C_{\boldsymbol{\theta}}\left[\sum_{\mathbf{q} \subseteq \boldsymbol{\theta}} \prod_{(i, j)=(1,0)}^{\left(N_{L}, N_{R}\right)} \psi_{i j}{ }^{q_{i j}} \prod_{i=1}^{N_{L}}\left(\begin{array}{c}
\theta_{i} \\
\mathbf{q}_{\mathbf{i} \bullet}
\end{array}\right)-1\right] \\
& =\frac{L_{0}}{K_{x}^{*}} \sum_{\boldsymbol{\theta} \in \Theta} C_{\boldsymbol{\theta}}\left[\left|\boldsymbol{\psi}_{\mathbf{1}} \bullet\right|^{\theta_{1}}\left|\boldsymbol{\psi}_{\mathbf{2}} \bullet\right|^{\theta_{2}} \ldots\left|\boldsymbol{\psi}_{\boldsymbol{N}_{\boldsymbol{L}} \bullet}\right|^{\theta_{N_{L}}-1}\right] \\
& =\frac{L_{0}}{K_{x}^{*}} \sum_{\boldsymbol{\theta} \in \Theta} C_{\boldsymbol{\theta}}\left[\prod_{i=1}^{N_{L}}\left|\boldsymbol{\psi}_{\boldsymbol{i}} \bullet\right|^{\theta_{i}}-1\right]
\end{aligned}
$$

when $\left|\boldsymbol{\psi}_{\boldsymbol{i} \bullet}\right|=\sum_{j=0}^{N_{R}} \psi_{i j}$, and the predicted amount of bound complex $\boldsymbol{\theta}$ (complex of each kind) is

$$
L_{\text {bound }, \boldsymbol{\theta}}=\frac{L_{0} C_{\boldsymbol{\theta}}}{K_{x}^{*}}\left[\prod_{i=1}^{N_{L}}\left|\boldsymbol{\psi}_{\boldsymbol{i}}\right|^{\theta_{i}}-1\right]
$$

The amount of fully bound ligands. In multivalent complexes like bispecific antibodies, drug activity may require that all subunits be bound to their respective 
targets [1]. The predicted amount of ligand full-valently bound can be calculated as

$$
\begin{aligned}
v_{\text {full,eq }} & =\sum_{\boldsymbol{\theta} \in \Theta} \sum_{q_{10}, \ldots, q_{N_{L} 0}=0} \frac{L_{0} C_{\boldsymbol{\theta}}}{K_{x}^{*}} \prod_{(i, j)=(1,1)}^{\left(N_{L}, N_{R}\right)} \psi_{i j}^{q_{i j}}\left(\begin{array}{c}
\theta_{1} \\
\mathbf{q}_{\mathbf{1} \bullet}^{*}
\end{array}\right) \ldots\left(\begin{array}{c}
\theta_{N_{L}} \\
\mathbf{q}_{\mathbf{N}_{\mathbf{L}}}^{*} \bullet
\end{array}\right) \\
& =\frac{L_{0}}{K_{x}^{*}} \sum_{\boldsymbol{\theta} \in \Theta} C_{\boldsymbol{\theta}} \prod_{i=1}^{N_{L}}\left(\sum_{j=1}^{N_{R}} \psi_{i j}\right)^{\theta_{i}} \\
& =\frac{L_{0}}{K_{x}^{*}} \sum_{\boldsymbol{\theta} \in \Theta} C_{\boldsymbol{\theta}} \prod_{i=1}^{N_{L}}\left(\left|\boldsymbol{\psi}_{\boldsymbol{i}}\right|-1\right)^{\theta_{i}},
\end{aligned}
$$

230 multinomial coefficient $\left(\begin{array}{c}\theta_{i} \\ \mathbf{q}_{\mathbf{i}}^{*}\end{array}\right)$ describes the number of ways one can allocate $\theta_{i}$ receptors to any position in the $i$-th row of the $\left(q_{i j}\right)$ matrix except the 0 -th row which stands for unbound.

In fact, the predicted amount of any specific-valently bound ligands can be derived in such manner. For example, the amount of ligands that bind monovalently can be calculated as

$$
\begin{aligned}
v_{1, \mathrm{eq}} & =\sum_{\boldsymbol{\theta} \in \Theta} \frac{L_{0} C_{\boldsymbol{\theta}}}{K_{x}^{*}} \sum_{i=1}^{N_{L}} \sum_{j=1}^{N_{R}} \psi_{i j}^{q_{i j}}\left(\begin{array}{c}
\theta_{i} \\
1
\end{array}\right) \\
& =\sum_{\boldsymbol{\theta} \in \Theta} \frac{L_{0} C_{\boldsymbol{\theta}}}{K_{x}^{*}} \sum_{i=1}^{N_{L}}\left|\boldsymbol{\psi}_{\boldsymbol{i}}\right| \theta_{i} .
\end{aligned}
$$

This can be used for estimating the amount of multimerized ligands, $L_{\text {multi }}=$ $L_{\text {bound }}-v_{1, \text { eq }}$, and multimerized receptors, $R_{\text {multi }}=R_{\text {bound }}-v_{1, \text { eq }}$.

\subsection{Complexes formed through random assortment}

Another common mode of forming multivalent complexes in biology, such as in the formation of antibody-antigen complexes [4, is the stochastic assembly of monomer units to a common scaffold. Instead of a specific arrangement, we 240 provide binding compounds of a fixed valency $f$ and a mixture of monomer ligands, and complexes can form through random assortment. The concentration of these complexes, therefore, will follow a multinomial distribution.

To formulate this mathematically, we denote the proportion of $L_{i}$ as $C_{i}$, and $\sum_{i=1}^{N_{L}} C_{i}=1$. For example, say we have $40 \% L_{1}$ and $60 \% L_{2}$ in the solution

245 to form dimers $(f=2)$, then $C_{1}=40 \%, C_{2}=60 \%$. As complex formation follows a binomial distribution, there will be $16 \%$ bivalent $L_{1}, 36 \%$ bivalent $L_{2}$, and $48 \% L_{1}-L_{2}$ complex. In general, the probability of complexes formed as described by $\boldsymbol{\theta}$ in random assortment is

$$
C_{\boldsymbol{\theta}}=\left(\begin{array}{c}
f \\
\boldsymbol{\theta}
\end{array}\right) C_{1}^{\theta_{1}} C_{2}^{\theta_{2}} \ldots C_{N_{L}}^{\theta_{N_{L}}}=\left(\begin{array}{c}
f \\
\boldsymbol{\theta}
\end{array}\right) \prod_{i=1}^{N_{L}} C_{i}^{\theta_{i}} .
$$


Since $\sum_{i=1}^{N_{L}} C_{i}=1$, we know that

$$
\sum_{\boldsymbol{\theta} \in \Theta} C_{\boldsymbol{\theta}}=\sum_{\boldsymbol{\theta} \in \Theta}\left(\begin{array}{l}
f \\
\boldsymbol{\theta}
\end{array}\right) \prod_{i=1}^{N_{L}} C_{i}^{\theta_{i}}=\left(C_{1}+C_{2}+\ldots+C_{N_{L}}\right)^{f}=1 .
$$

250

Plugging this relationship between $C_{\boldsymbol{\theta}}$ and $C_{i}$ into Equation 3.6 for the amount of a specific binding configuration, we have

$$
\begin{aligned}
v_{\mathbf{q}} & =\frac{L_{0}}{K_{x}^{*}}\left(\begin{array}{c}
f \\
\boldsymbol{\theta}(\mathbf{q})
\end{array}\right) \prod_{i=1}^{N_{L}} C_{i}^{\theta_{i}(\mathbf{q})} \prod_{(i, j)=(1,1)}^{\left(N_{L}, N_{R}\right)} \psi_{i j}^{q_{i j}} \prod_{i=1}^{N_{L}}\left(\begin{array}{c}
\theta_{i}(\mathbf{q}) \\
\mathbf{q}_{\mathbf{i} \bullet}
\end{array}\right) \\
& =\frac{L_{0}}{K_{x}^{*}}\left(\begin{array}{l}
f \\
\mathbf{q}
\end{array}\right) \prod_{(i, j)=(1,0)}^{\left(N_{L}, N_{R}\right)} C_{i}^{q_{i j}} \prod_{(i, j)=(1,1)}^{\left(N_{L}, N_{R}\right)} \psi_{i j}^{q_{i j}} \\
& =\frac{L_{0}}{K_{x}^{*}}\left(\begin{array}{l}
f \\
\mathbf{q}
\end{array}\right) \prod_{(i, j)=(1,0)}^{\left(N_{L}, N_{R}\right)} \varphi_{i j}^{q_{i j}},
\end{aligned}
$$

where $\varphi_{i j}=R_{\mathrm{eq}, j} K_{a, i j} K_{x}^{*} C_{i}$ and $\varphi_{i 0}=C_{i}$.

Solve the amount of free receptors. Similar to the specific arrangement case, we still need to solve $R_{\text {eq, } n}$ numerically from $R_{\text {tot }, n}$. We first derive the amount of bound receptors of each kind at equilibrium as

$$
\begin{aligned}
R_{\text {bound }, n} & =\sum_{\mathbf{q} \in Q}\left|\mathbf{q}_{\bullet \mathbf{n}}\right| v_{\mathbf{q}} \\
& =\sum_{\mathbf{q} \in Q}|\mathbf{q} \bullet \mathbf{n}|\left(\begin{array}{l}
f \\
\mathbf{q}
\end{array}\right) \frac{L_{0}}{K_{x}^{*}} \prod_{(i, j)=(1,0)}^{\left(N_{L}, N_{R}\right)} \varphi_{i j}^{q_{i j}} \\
& =\frac{L_{0}}{K_{x}^{*}}\left|\varphi_{\bullet n}\right| f\left[\sum_{(i, j)=(1,0)}^{\left(N_{L}, N_{R}\right)}\right]_{i j}^{f-1} \\
& =\frac{L_{0} f}{K_{x}^{*}}\left|\varphi_{\bullet n}\right|(1+\Phi)^{f-1} .
\end{aligned}
$$

Then by the conservation of mass, we can numerically solve $R_{\text {eq }, n}$ as

$$
\begin{aligned}
R_{\mathrm{tot}, n} & =R_{\mathrm{eq}, n}+R_{\mathrm{bound}, n} \\
& =R_{\mathrm{eq}, n}+\frac{L_{0} f}{K_{x}^{*}}\left|\boldsymbol{\varphi}_{\bullet n}\right|(1+\Phi)^{f-1} .
\end{aligned}
$$

Again, since $\Phi=\sum_{j=1}^{N_{R}}\left|\varphi_{\bullet j}\right|$ is a function of every $R_{\text {eq }, n}$, all $R_{\text {eq }, n}$ need to be solved together. 
The amount of $k$-valently bound complexes. For randomly assorted complexes, we first derive the amount of ligands that bind $k$-valently. As we will show, it has a useful expression that can used to find many other quantities conveniently. First, let's break $\mathbf{q}$ into two separate vectors, $\mathbf{q}=(\mathbf{q} \bullet \mathbf{0}, \mathbf{q} \cdot \mathbf{* x})$. We define the vector formed by the 0 -th column of $\mathbf{q}$ which stand for unbound as $\mathbf{q} \cdot \mathbf{0}$, and the one formed by the other elements as $\mathbf{q} \cdot \mathbf{x}$. By the model setup, $|\mathbf{q}|=f$, $|\mathbf{q} \bullet \mathbf{x}|=k$, and $|\mathbf{q} \bullet \mathbf{0}|=f-k$. We then have

$$
\begin{aligned}
& v_{k, \text { eq }}=\sum_{\substack{|\mathbf{q} \bullet x\\
| \mathbf{q} \bullet \mathbf{*} \mid=k-k}} v_{\mathbf{q}}=\sum_{\substack{|\mathbf{q} \bullet x|=k \\
|\mathbf{q} \cdot \mathbf{0}|=f-k}}\left(\begin{array}{cc}
f \\
\mathbf{q} \bullet \mathbf{* x} & \mathbf{q} \bullet \mathbf{\bullet 0}
\end{array}\right) \frac{L_{0}}{K_{x}^{*}} \prod_{(i, j)=(1,0)}^{\left(N_{L}, N_{R}\right)} \varphi_{i j}{ }^{q_{i j}} \\
& =\sum_{\substack{|\mathbf{q} \cdot \mathbf{*}|=k \\
|\mathbf{q} \bullet \mathbf{0}|=f-k}}\left(\begin{array}{c}
f \\
k
\end{array}\right)\left(\begin{array}{c}
k \\
\mathbf{q} \bullet \mathbf{x}
\end{array}\right)\left(\begin{array}{c}
f-k \\
\mathbf{q} \bullet \mathbf{0}
\end{array}\right) \frac{L_{0}}{K_{x}^{*}} \prod_{(i, j)=(1,1)}^{\left(N_{L}, N_{R}\right)} \varphi_{i j} q_{i j} \prod_{i=1}^{i=N_{L}} C_{i}^{q_{i 0}} \\
& =\frac{L_{0}}{K_{x}^{*}}\left(\begin{array}{l}
f \\
k
\end{array}\right)\left[\sum_{|\mathbf{q} \bullet \mathbf{x}|=k}\left(\begin{array}{c}
k \\
\mathbf{q} \bullet \mathbf{x}
\end{array}\right) \prod_{(i, j)=(1,1)}^{\left(N_{L}, N_{R}\right)} \varphi_{i j} q_{i j}\right]\left[\sum_{|\mathbf{q} \bullet \mathbf{o}|=f-k}\left(\begin{array}{c}
f-k \\
\mathbf{q} \bullet \mathbf{0}
\end{array}\right) \prod_{i=1}^{i=N_{L}} C_{i}{ }^{q_{i 0}}\right] \\
& =\frac{L_{0}}{K_{x}^{*}}\left(\begin{array}{l}
f \\
k
\end{array}\right)\left[\sum_{(i, j)=(1,1)}^{\left(N_{L}, N_{R}\right)} \varphi_{i j}{ }^{q_{i j}}\right]^{k}\left[\sum_{i=1}^{i=N_{L}} C_{i}\right]^{f-k}=\frac{L_{0}}{K_{x}^{*}}\left(\begin{array}{l}
f \\
i
\end{array}\right) \Phi^{k} .
\end{aligned}
$$

The amount of total bound ligands and receptors. Many macroscopic properties can be derived from $v_{k \text {,eq }}$. For example, the amount of total bound ligands is simply the sum of ligands bound monovalently to fully, and can be simplified to

$$
\begin{aligned}
L_{\mathrm{bound}} & =\sum_{k=1}^{f} v_{k, \mathrm{eq}}=\sum_{k=0}^{f} v_{k, \mathrm{eq}}-v_{0, \mathrm{eq}} \\
& =\sum_{k=0}^{f} \frac{L_{0}}{K_{x}^{*}}\left(\begin{array}{l}
f \\
k
\end{array}\right) \Phi^{k}-\frac{L_{0}}{K_{x}^{*}}\left(\begin{array}{l}
f \\
0
\end{array}\right) \Phi^{0} \\
& =\frac{L_{0}}{K_{x}^{*}}\left[(1+\Phi)^{f}-1\right] .
\end{aligned}
$$

Similarly, the total bound receptors should be

$$
\begin{aligned}
R_{\text {bound }} & =\sum_{k=1}^{f} k v_{k, \text { eq }}=\sum_{k=1}^{f} k \frac{L_{0}}{K_{x}^{*}}\left(\begin{array}{l}
f \\
k
\end{array}\right) \Phi^{k} \\
& =\frac{L_{0}}{K_{x}^{*}} f \Phi(1+\Phi)^{f-1} .
\end{aligned}
$$

As we show here, these quantities all have elegant closed form solutions, and they are only dependent on $\Phi$, a single value that incorporate all information 
about receptor amounts, monomer ligand compositions, and binding affinities. $\Phi$ was previously defined as $\Phi=\sum_{i=1}^{N_{L}} \sum_{j=1}^{N_{R}} \varphi_{i j}=\sum_{i=1}^{N_{L}} \sum_{j=1}^{N_{R}} R_{\mathrm{eq}, j} K_{a, i j} K_{x}^{*} C_{i}$.

The number of cross-linked receptors. In some biological contexts such as T cell receptor-MHC [3] or antibody-Fc receptor [4] interactions, signal transduction is driven by receptor cross-linking due to multivalent binding. The amount of total cross-linked receptors can be derived from $v_{k, \text { eq }}$ as

$$
\begin{aligned}
R_{\text {multi }} & =\sum_{k=2}^{f} k v_{k, \text { eq }}=R_{\text {bound }}-v_{1, \text { eq }} \\
& =\frac{L_{0}}{K_{x}^{*}} f \Phi(1+\Phi)^{f-1}-\frac{L_{0}}{K_{x}^{*}}\left(\begin{array}{l}
f \\
1
\end{array}\right) \Phi=\frac{L_{0}}{K_{x}^{*}} f \Phi\left[(1+\Phi)^{f-1}-1\right] .
\end{aligned}
$$

To find the number of crosslinked receptors of a specific kind, $R_{n}$, requires extra consideration. Similar to how $v_{k \text {,eq }}$ was found, we break $\mathbf{q}$ into three separate vectors, $\mathbf{q}=\left(\mathbf{q}_{\bullet 0}, \mathbf{q}_{\bullet \mathbf{n}}, \mathbf{q}_{\bullet \mathbf{x}}\right)$. $\mathbf{q}_{\bullet 0}$ is the vector formed by the 0 -th column of $\mathbf{q}$, q.n is the vector formed by the $n$-th column of $\mathbf{q}$, and $\mathbf{q} \bullet \mathbf{x}$ contains all others. If the complex is $s$-valently bound, then $\left|\mathbf{q}_{\bullet 0}\right|=f-s$. We further assume that $\left|\mathbf{q}_{\mathbf{\prime}}\right|=t$, then $\left|\mathbf{q}_{\mathbf{* x}}\right|=s-t$. By this setup, we have

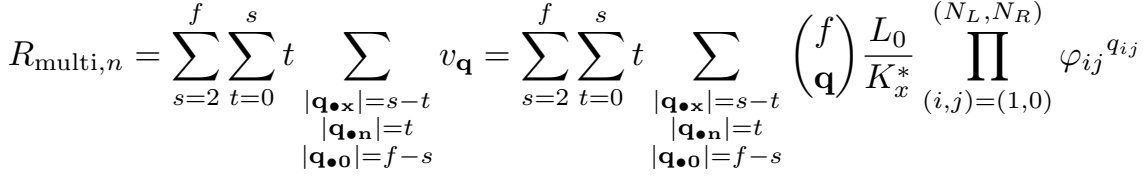

$$
\begin{aligned}
& =\sum_{s=2}^{f} \sum_{t=0}^{s} \frac{t L_{0}}{K_{x}^{*}}\left(\begin{array}{ccc} 
& f \\
s-t & t & f-s
\end{array}\right)\left[\sum_{|\mathbf{q} \bullet \mathbf{*}|=s-t}\left(\begin{array}{c}
s-t \\
\mathbf{q} \cdot \mathbf{* x}
\end{array}\right) \prod_{\substack{i, j)=(1,0) \\
j \neq n}}^{\left(N_{L}, N_{R}\right)} \varphi_{i j}{ }^{q_{i j}}\right] \\
& {\left[\sum_{|\mathbf{q} \bullet \mathbf{n}|=t}\left(\begin{array}{c}
t \\
\mathbf{q} \bullet \mathbf{n}
\end{array}\right) \prod_{i=1}^{N_{L}} \varphi_{i n}{ }^{q_{i n}}\right]\left[\sum_{|\mathbf{q} \bullet|=f-s}\left(\begin{array}{c}
f-s \\
\mathbf{q} \bullet \mathbf{0}
\end{array}\right) \prod_{i=1}^{N_{L}} C_{i}^{q_{i 0}}\right]} \\
& =\sum_{s=2}^{f} \sum_{t=0}^{s} \frac{t L_{0}}{K_{x}^{*}}\left(\begin{array}{ccc} 
& f & \\
s-t & t & f-s
\end{array}\right)\left(\Phi-\left|\varphi_{\bullet}\right|\right)^{s-t}\left|\varphi_{\bullet n}\right|^{t}\left(\sum_{i=1}^{N_{L}} C_{i}\right)^{f-s} \\
& =\sum_{s=2}^{f} \frac{L_{0}}{K_{x}^{*}}\left[\sum_{t=0}^{s} t\left(\begin{array}{l}
s \\
t
\end{array}\right)\left(\frac{\left|\varphi_{\bullet n}\right|}{\Phi-\left|\varphi_{\bullet n}\right|}\right)^{t}\right]\left(\begin{array}{l}
f \\
s
\end{array}\right)\left(\Phi-\left|\varphi_{\bullet n}\right|\right)^{s} \\
& =\sum_{s=2}^{f} \frac{L_{0}}{K_{x}^{*}} s\left(\frac{\left|\varphi_{\bullet n}\right|}{\Phi-\left|\varphi_{\bullet n}\right|}\right)\left(\frac{\Phi}{\Phi-\left|\varphi_{\bullet n}\right|}\right)^{s-1}\left(\begin{array}{l}
f \\
s
\end{array}\right)\left(\Phi-\left|\varphi_{\bullet n}\right|\right)^{s} \\
& =\frac{L_{0}}{K_{x}^{*}}\left[\sum_{s=2}^{f} s\left(\begin{array}{l}
f \\
s
\end{array}\right) \Phi^{s}\right] \frac{\left|\varphi_{\bullet}\right|}{\Phi}
\end{aligned}
$$




$$
\begin{aligned}
& =\frac{L_{0}}{K_{x}^{*}} \frac{\left|\varphi_{\bullet}\right|}{\Phi}\left[f \Phi(1+\Phi)^{f-1}-f \Phi\right] \\
& =\frac{L_{0} f}{K_{x}^{*}}\left|\varphi_{\bullet}\right|\left[(1+\Phi)^{f-1}-1\right] .
\end{aligned}
$$

This formula can useful when investigating the role of each receptor in a tial ligand concentration, and $L_{\text {init, } \boldsymbol{\theta}}$ is the initial concentration of complex $\boldsymbol{\theta}$. $L_{\text {bound }}$ and $L_{\text {bound, } \boldsymbol{\theta}}$ may be found at Equations 4.14 and 4.5 , depending on the occasion. (4) Finally, certain aspects of the system may not be sensitive to ligand concentration as an input parameter, or one could treat concentration as an unknown. 


\section{Application examples}

In previous sections, we have shown how all macroscopic predictions made in this work can be written in closed form formulae. Therefore, some computationally expensive analyses are enabled by the efficiency of our model. Here, we provide two examples to demonstrate the utility of large-scale predictions made possible by this model.

\subsection{Mixture binding prediction}

Leveraging a synergistic effect among two or more drugs is of great interest in pharmaceutical development. A challenge in investigating synergy is to identify its underlying source. Most biological pathways follow a similar pattern: when the drug binds to certain surface receptors of a cell, a downstream pathway in the cell is initiated, leading to some actions. Therefore in general, synergism can come from either the initial binding events themselves or downstream signal transduction interactions. Binding-level synergy means that merely using a combination of ligands boosts the amount of binding to the important recep335 tors and thus intensifies the overall effect. Downstream effect synergy indicates that the benefit of using mixtures arises from other cellular regulatory mechanisms two ligands can bring about. The binding model we propose here can help to investigate this issue by offering accurate predictions for the binding of multivalent complex mixtures.
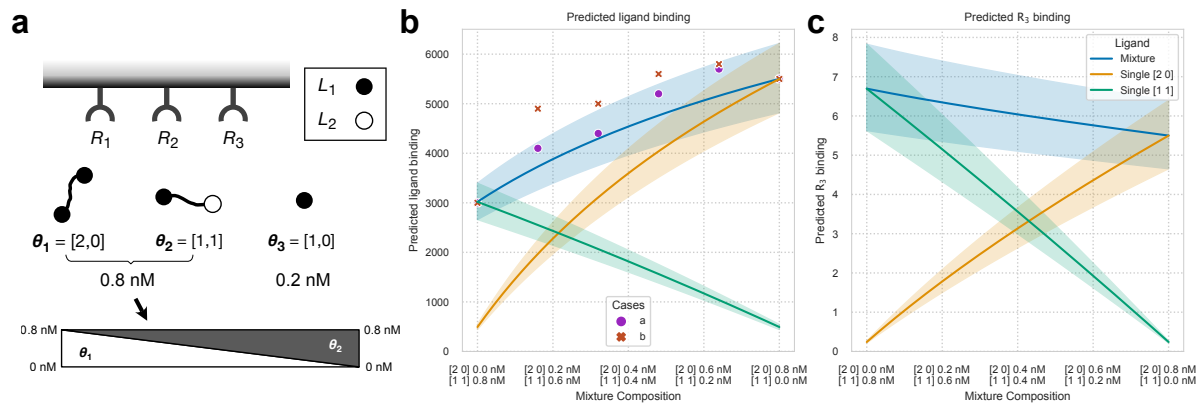

Figure 3: Prediction on mixture binding of $\boldsymbol{\theta}_{\mathbf{1}}=[2,0], \boldsymbol{\theta}_{\mathbf{2}}=[1,1]$, and $\boldsymbol{\theta}_{\mathbf{3}}=[1,0]$. (a) $\mathrm{A}$ schematic of the binding scenario; (b) The predicted total ligand binding; (c) The amount of bound $R_{3}$ at equilibrium. Shaded areas in (b), (c) are simulated confidence intervals by varying the receptor levels up and down by $10 \%$. The points on (b) represent possible experimental results, arbitrarily drawn for demonstration. In case $a$ (purple circles), since most data points are inside the confidence interval, we can assume the measurement error can explain these variations. In case $b$ (orange crosses), however, the synergism of these complexes are beyond the binding level.

In Figure 3, we provide an example of mixture binding predictions (Figure 3a). We investigate a mixture of three types of ligand complexes, bivalent $L_{1}$ $\left(\boldsymbol{\theta}_{\mathbf{1}}=[2,0]\right)$, bispecific $L_{1}-L_{2}\left(\boldsymbol{\theta}_{\mathbf{2}}=[1,1]\right)$, and monovalent $L_{1}\left(\boldsymbol{\theta}_{\mathbf{3}}=[1,0]\right)$. The crosslinking constant is set to be $K_{x}^{*}=10^{-12}$ cell $\cdot \mathrm{M}$, similar to previous results [4. We predict the amount of binding of this mixture to a cell expressing 
three types of receptors, with $\boldsymbol{R}_{\mathrm{tot}}=\left[2.5 \times 10^{4}, 3 \times 10^{4}, 2 \times 10^{3}\right]$ cell $^{-1}$. The affinity constants of $L_{1}$ to these three receptors are $\boldsymbol{K}_{\boldsymbol{a}, \mathbf{1} \boldsymbol{\bullet}}=\left[1 \times 10^{8}, 1 \times\right.$ $\left.10^{5}, 6 \times 10^{5}\right] \mathrm{M}^{-1}$, and of $L_{2}, \boldsymbol{K}_{\boldsymbol{a}, \mathbf{2} \bullet}=\left[3 \times 10^{5}, 1 \times 10^{7}, 1 \times 10^{6}\right] \mathrm{M}^{-1}$. Here, we investigate the changing concentration of $\boldsymbol{\theta}_{\mathbf{1}}$ and $\boldsymbol{\theta}_{\mathbf{2}}$, while holding the amount of $\boldsymbol{\theta}_{3}$ constant at $0.2 \mathrm{nM}$. Figure 3 shows the predicted total ligand bound 350 (Figure $3 \mathrm{~b}$ ) and $R_{3}$ bound (Figure $3 \mathrm{c}$ ) for only $\boldsymbol{\theta}_{\mathbf{1}}$ or $\boldsymbol{\theta}_{\mathbf{2}}$ with concentration from 0 to $0.8 \mathrm{nM}$, and their mixtures in every possible composition with total concentration $0.8 \mathrm{nM}$.

Mixture binding prediction can help us identify the source of synergy. To connect model predictions to experimental measurements, ligand binding might be measured by fluorescently-tagged ligands, while the number of bound receptors of a specific type might associate with an indirect measurement such as cellular response. After making a series of measurements for different compositions of mixtures, we can fit the $100 \%$ of one complex cases (numbers on the two ends on the plot) first and then compare the mixture measurements to the predictions. Determining whether the downstream effect contributes to the observed synergy (or antagonism) can be framed as a hypothesis testing problem:

\section{$\boldsymbol{H}_{\mathbf{0}}$ : The synergism of the mixture can be explained solely by binding.}

The uncertainty of mixture binding prediction comes from measurement errors of receptor abundance and binding affinities. Usually, the receptor expression of a cell population has an empirical distribution which can be measured. The confidence intervals in Figure 3 were drawn with the assumption that receptor expression fluctuates up and down by $10 \%$ (coinciding with log-normally distributed amounts). Also, due to the measurement technique, the binding affinities may be over- or underestimated [12. The confidence interval of mixture prediction can be determined by the model with all these considered, and a $p$-value can be derived.

We arbitrarily drew some possible experimental results on Figure $3 \mathrm{~b}$ for demonstration. If most mixture measurements fall within the confidence in375 terval of the predictions (such as case $a$ annotated by the purple circles in Figure $3 \mathrm{~b}$ ), the synergy will very likely come from binding only. However, if the measurements are obviously beyond the confidence interval (case $b$, the orange crosses), it is reasonable to suspect a synergistic (or antagonistic) effect beyond binding alone. With the flexibility of the binding model, this method can also be extended to a mixture of more than two compounds.

\subsection{Binding space of a ligand}

When a dose of ligand (drug, hormone, cytokine, etc.) is released into the circulation system of an individual due to either physiological response or exogenous administration, the compound will spread and bind to many cell populations to varying extents. An essential question in pharmacology is how much a compound will bind to their intended target populations compared to offtarget ones. This question is important for understanding basic biology as well 
as developing new therapeutics. For example, hormones and cytokines are important signaling molecules, and having a quantitative prediction of on- and off-target binding can help us understand their mechanism greatly. For drug development, binding prediction can guide optimization to improve specificity toward the intended targets [13. A cell population can be defined by the receptors they express. Therefore, given the parameters of the dose and the receptor profile of each cell population, our model can make all the predictions discussed previously.

From the perspective of this binding model, there is nothing special about any specific cell population. If the local concentration is constant everywhere, our model can map any cell with a certain receptor expression to the amount of binding induced by this dose. If the biological activity of this compound on a cell is related to the quantity of binding to a certain ligand or receptor, the effect of this dose can be written as a function $f$, with

$$
\boldsymbol{R}_{\text {tot }} \in \mathbb{R}_{+}^{N_{R}} \mapsto f\left(\boldsymbol{R}_{\text {tot }}\right) \in \mathbb{R}_{+},
$$

where $\boldsymbol{R}_{\text {tot }}$ is a vector of nonnegative entries that describes the cell's expression of $N_{R}$ receptors, and $f\left(\boldsymbol{R}_{\text {tot }}\right)$ is the amount of binding. Here, we define the binding behavior of this dose (or any compound) as its binding space.

a

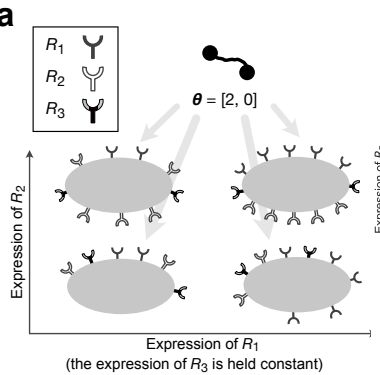

b

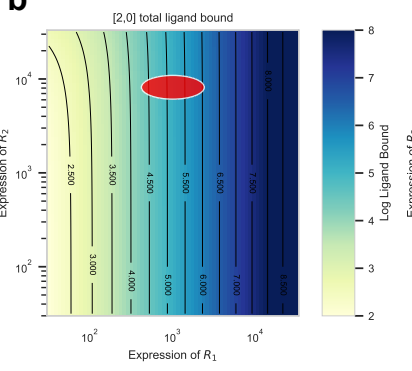

C $1201 R_{2}$ bound

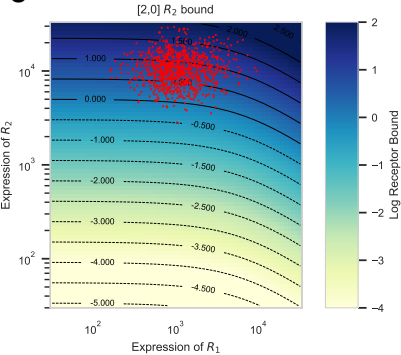

Figure 4: The binding space of $1 \mathrm{nM} \boldsymbol{\theta}=[2,0]$. (a) A schematic diagram of the ligand and four examples of receptor-expressing cells represented by the coordinate; (b) The amount of total ligand bound; (c) receptor $R_{2}$ bound predictions. The $\mathrm{x}$ - and y-axis show the expression of $R_{1}$ and $R_{2}$, while the expression of $R_{3}$ is a constant, $2.0 \times 10^{3}$ cell $^{-1}$, and not shown. Any cell population can be drawn on the binding space. For example, the red ellipse on (b) represents a cell population with receptor expression of roughly $\boldsymbol{R}_{\text {tot }}=[1.0,10.0,2.0] \times 10^{3} \mathrm{cell}^{-1}$. We can alternatively project points of experimental single cell expression data onto a binding space, as shown on (c) (the points were generated arbitrarily assuming a population of log-normally distributed $R_{1}$ and $R_{2}$ expression for demonstration purpose.

In Figure 4, we plot the binding space of a bivalent $L_{1}$ ligand $\boldsymbol{\theta}=[2,0]$ with concentration $1 \mathrm{nM}$. The binding affinities are the same as described in the last subsection (5.1). In this binding space, we consider three receptors, $R_{1}, R_{2}$, and $R_{3}$. We plot how the amount of binding relates to the cell expression profile, $\boldsymbol{R}_{\text {tot }}$. Here, the amount of $R_{1}$ and $R_{2}$ varies with the two axes, while $R_{3}$ is ${ }_{410}$ held constant at $2.0 \times 10^{3}$ cell $^{-1}$. In this plot, each point represents a cell with a distinct expression profile, as some examples drawn on Figure 4a. Then we 
use colors and contour lines to show the amount of binding. From these two plots, we can see that although both ligand binding and $R_{2}$ binding increase with more receptors, ligand binding is more sensitive to $R_{1}$ amounts, and $R_{2}$ binding $R_{2}$ amounts. To consider any specific cell population, one only needs to determine where its expression profile falls on the plot and read the predictions from the contour line. For example, on Figure $4 \mathrm{~b}$, the red cell population will have about $e^{5.2}=181$ bound ligands per cell. The number of contour lines a population ride on can also show intrapopulation variation. In this case, we ${ }_{420}$ expect the variation in ligand binding to fall between $e^{4.3}=74$ and $e^{6.0}=403$.

The binding space can provide ample information about the compound. It is an intrinsic property of a ligand given its concentration and other ligand it mixes with, independent of any specific cell. The biological process of drug diffusion to a certain cell is analogous to sampling a point from this binding space. Its gradient indicates in which direction the binding level increases the fastest, as well as to which receptor amount it is the most sensitive. An inactive antagonist that introduces binding competition with the ligand can distort its binding space, and we can visualize it by the change of shape in the contour lines. This plot can also intuitively demonstrate intrapopulation binding variance and ${ }_{430}$ interpopulation cell specificity of the compound. With the development of highthroughput single-cell methods such as flow cytometry, the expression profiles of a collection of cells can be identified en masse, and we can overlap their results onto a binding space plot (as in Figure 4c). This shows the promise of applying our model to single-cell data. Although we can only visualize two receptors ${ }_{435}$ in a plot, binding space applies to any $N_{R}$ types of receptors. Theoretically, the concept of the binding space of a ligand is only complete when all relevant surface receptors are considered.

\section{Discussion}

In this work, we propose a mechanistic multivalent binding model that accounts for the interaction among multiple receptors and a mixture of ligand complexes formed by binding monomers. The flexible framework allows a mixture of both homogeneous and heterogeneous ligand complexes, even when they don't have the same valency. We first derive the amount of ligand of a specific binding configuration at equilibrium through the law of mass action. Using this formula, we make macroscopic predictions by applying the multinomial theorem strategically. Our predictions cover cases where complexes are formed by specific arrangement or random assortment. Finally, we provide two practical examples of how this model can help with biological research.

Compared with previous approaches, the model here is a uniquely scalable ${ }_{450}$ and elegant approach to multivalent binding when considering multivalent complexes of heterogeneous monomer composition and/or multiple receptors. Scalability to higher valency complexes is essential as rule-based computational models become impractical due to a combinatorial explosion of binding states. By contrast, our model can make a large number of predictions easily, enabling mixture synergy analysis and binding space calculations across individual cells. 
The mathematical elegance of the model welcomes analytical studies and incorporating it into higher-level computational frameworks. For example, we apply auto-differentiation to ensure accuracy in the root-finding operation when solving for unbound receptor. We have similarly used auto-differentiation to solve for the gradients of the model with respect to input quantities when fitting it to data points. One could even feasibly derive analytical forms of the gradients. This enables one to build more complex computational models on top of this binding framework, such as inferring the composition of multivalent complexes in solution from indirect high-throughput assays. While differentiation of dif465 ferential equation models is possible through adjoint state methods, solving can be sensitive to the parameters of the system, is much less efficient, and requires trade-offs in accuracy for performance.

The assumptions made in this model may compromise its accuracy in some cases. Our setup has a single crosslinking constant, $K_{x}^{*}$, to reflect the multivalency effect. In practice, this model has worked well in predicting experimental binding results [13, 14, 4]. However, the steric effects of a multivalent ligand can be more complicated and context-dependent. The complication of multivalency effect comes from the geometry of ligand complexes that introduced steric effect as well as the distribution of receptors on the cell surface. For instance, the 475 length of the hinge region is needed to estimate the radius of area a molecule can reach [15]. Receptor clustering can be play a big role in the behavior of ligand binding as well [16]. Accounting for these effects requires more in-depth studies than just measuring the monomer binding kinetics. Some other computational approaches investigate steric effects more meticulously, but inevitably introduce some added complexity. For example, previous work has conducted a case-by-case exploration of how ligands bind when distributed randomly or ordered, arranged as a lattice, ring, or chain to give a better hindrance factor estimation [10. When the actual situation is not known, our model can serve as an adequate starting point.

485 Although this model is very general purpose, it mainly focuses on the binding dynamics on a cell surface, similar to the previous work on which it is based [7, 8, 5]. For ligands discordant with the two-step binding process shown in Figure 2 , other model constructions might be necessary. For example, some previous work focuses on scaffold proteins as intracellular multivalent complexes [17, but these often lack independence between the individual monomer binding events. In this case, various alternative computational models have been developed [18, 19, 20].

Surface receptor binding is a universal event in biology. A prevalent question calls for a general solution. We expect this model to be successfully applied

495 to many contexts. Previously, we have used a simpler version of the random assortment model to accurately predict IgG antibody-Fc $\gamma \mathrm{R}$ interactions [4], and also applied it to fit epithelial cell adhesion molecule binding data [13, 14]. We are also working on applying the model to IL-2 immunocomplexes [21, for optimization of high-valency cytokines with specific cell targeting [22], design 500 of cytokine-antibody bispecific antibody fusions, and as a factorization kernel in dimensionality reduction of systems serology data 23. With the arise of 
multispecific drugs in the recent decade [24, we expect this model to apply even more widely, exhibit its full competence and facilitate both basic scientific research and new therapy development. A.S.M. The authors declare no competing financial interests.

Author contributions. Z.C.T.: Methodology, Writing - original draft; A.S.M.: Funding acquisition, Writing - review \& editing.

Data and software availability. A Python package of this model and the code

\section{References}

[1] J. M. Paar, N. T. Harris, D. Holowka, B. Baird, Bivalent ligands with rigid double-stranded DNA spacers reveal structural constraints on signaling by FceRI, The Journal of Immunology 169 (2) (2002) 856-864.

[2] W. S. Hlavacek, A. S. Perelson, B. Sulzer, J. Bold, J. Paar, W. Gorman, R. G. Posner, Quantifying aggregation of IgE-FceRI by multivalent antigen, Biophysical journal 76 (5) (1999) 2421-2431.

[3] J. D. Stone, J. R. Cochran, L. J. Stern, T-cell activation by soluble MHC oligomers can be described by a two-parameter binding model, Biophysical Journal 81 (5) (2001) 2547-2557.

[4] R. A. Robinett, N. Guan, A. Lux, M. Biburger, F. Nimmerjahn, A. S. Meyer, Dissecting Fc $\gamma \mathrm{R}$ regulation through a multivalent binding model, Cell systems 7 (1) (2018) 41-48.

[5] A. S. Perelson, Receptor clustering on a cell surface. III. theory of receptor cross-linking by multivalent ligands: description by ligand states, Mathematical Biosciences 53 (1-2) (1981) 1-39.

[6] W. J. Errington, B. Bruncsics, C. A. Sarkar, Mechanisms of noncanonical binding dynamics in multivalent protein-protein interactions, Proceedings of the National Academy of Sciences 116 (51) (2019) 25659-25667.

[7] A. S. Perelson, C. DeLisi, Receptor clustering on a cell surface. I. theory of receptor cross-linking by ligands bearing two chemically identical functional groups, Mathematical Biosciences 48 (1-2) (1980) 71-110.

[8] A. S. Perelson, Receptor clustering on a cell surface. II. theory of receptor cross-linking by ligands bearing two chemically distinct functional groups, Mathematical Biosciences 49 (1) (1980) 87-110. 
[9] C. A. Macken, A. S. Perelson, Branching processes applied to cell surface aggregation phenomena, Vol. 58, Springer Science \& Business Media, 2013.

[10] W. S. Hlavacek, R. G. Posner, A. S. Perelson, Steric effects on multivalent ligand-receptor binding: exclusion of ligand sites by bound cell surface receptors, Biophysical journal 76 (6) (1999) 3031-3043.

[11] E. C. Piccione, S. Juarez, J. Liu, S. Tseng, C. E. Ryan, C. Narayanan, L. Wang, K. Weiskopf, R. Majeti, A bispecific antibody targeting CD47 and CD20 selectively binds and eliminates dual antigen expressing lymphoma cells, in: MAbs, Vol. 7(5), Taylor \& Francis, 2015, pp. 946-956.

[12] S. Hunter, J. Cochran, Cell-binding assays for determining the affinity of protein-protein interactions: technologies and considerations, Methods in enzymology 580 (2016) 21-44.

[13] Z. C. Tan, B. Orcutt-Jahns, A. S. Meyer, A quantitative view of strategies to engineer cell-selective ligand binding, bioRxiv (2020).

[14] C. M. Csizmar, J. R. Petersburg, T. J. Perry, L. Rozumalski, B. J. Hackel, C. R. Wagner, Multivalent ligand binding to cell membrane antigens: defining the interplay of affinity, valency, and expression density, Journal of the American Chemical Society 141 (1) (2018) 251-261.

[15] J. J. Rhoden, G. L. Dyas, V. J. Wroblewski, A modeling and experimental investigation of the effects of antigen density, binding affinity, and antigen expression ratio on bispecific antibody binding to cell surface targets, Journal of Biological Chemistry 291 (21) (2016) 11337-11347.

[16] B. R. Caré, H. A. Soula, Impact of receptor clustering on ligand binding, BMC Systems Biology 5 (1) (2011) 1-13.

[17] S. A. Chapman, A. R. Asthagiri, Quantitative effect of scaffold abundance on signal propagation, Molecular systems biology 5 (1) (2009) 313.

[18] J. Yang, W. S. Hlavacek, Scaffold-mediated nucleation of protein signaling complexes: elementary principles, Mathematical biosciences 232 (2) (2011) 164-173.

[19] A. Levchenko, J. Bruck, P. W. Sternberg, Scaffold proteins may biphasically affect the levels of mitogen-activated protein kinase signaling and reduce its threshold properties, Proceedings of the National Academy of Sciences 97 (11) (2000) 5818-5823.

[20] Y. Wu, J. Vendome, L. Shapiro, A. Ben-Shaul, B. Honig, Transforming binding affinities from three dimensions to two with application to cadherin clustering, Nature 475 (7357) (2011) 510-513. 
[21] J. B. Spangler, J. Tomala, V. C. Luca, K. M. Jude, S. Dong, A. M. Ring, P. Votavova, M. Pepper, M. Kovar, K. C. Garcia, Antibodies to interleukin2 elicit selective $\mathrm{T}$ cell subset potentiation through distinct conformational mechanisms, Immunity 42 (5) (2015) 815-825.

[22] B. Orcutt-Jahns, P. C. Emmel, E. M. Snyder, C. Posner, S. M. Carlson, A. S. Meyer, Multivalency enhances the specificity of Fc-cytokine fusions, bioRxiv (2021).

[23] A. W. Chung, M. P. Kumar, K. B. Arnold, W. H. Yu, M. K. Schoen, L. J. Dunphy, T. J. Suscovich, N. Frahm, C. Linde, A. E. Mahan, et al., Dissecting polyclonal vaccine-induced humoral immunity against HIV using systems serology, Cell 163 (4) (2015) 988-998.

[24] R. J. Deshaies, Multispecific drugs herald a new era of biopharmaceutical innovation, Nature 580 (7803) (2020) 329-338. 